\title{
Numerical Simulation of Wind Turbine Rotors Autorotation by Using the Modified LS-STAG Immersed Boundary Method
}

\author{
Ilia K. Marchevsky and Valeria V. Puzikova \\ Applied Mathematics Department, Bauman Moscow State Technical University (BMSTU), 5 2nd Baumanskaya, \\ Moscow 105005, Russia \\ Correspondence should be addressed to Ilia K. Marchevsky; iliamarchevsky@mail.ru
}

Received 7 April 2017; Revised 21 July 2017; Accepted 14 August 2017; Published 11 October 2017

Academic Editor: Lin Zhu

Copyright ( 2017 Ilia K. Marchevsky and Valeria V. Puzikova. This is an open access article distributed under the Creative Commons Attribution License, which permits unrestricted use, distribution, and reproduction in any medium, provided the original work is properly cited.

\begin{abstract}
A software package is developed for numerical simulation of wind turbine rotors autorotation by using the modified LS-STAG level-set/cut-cell immersed boundary method. The level-set function is used for immersed boundaries description. Algorithm of level-set function construction for complex-shaped airfoils, based on Bézier curves usage, is proposed. Also, algorithm for the level-set function recalculation at any time without reconstructing the Bézier curve for each new rotor position is described. The designed second-order Butterworth low-pass filter for aerodynamic torque filtration for simulations using coarse grids is presented. To verify the modified LS-STAG method, the flow past autorotating Savonius rotor with two blades was simulated at $\operatorname{Re}=1.96 \cdot 10^{5}$.
\end{abstract}

\section{Introduction}

The rotor is the first element in the chain of functional elements of a wind turbine. Its aerodynamic and dynamic properties, therefore, have a decisive influence on the entire system in many respects. The designer faces the problem of finding the relationship between the actual shape of the rotor, for example, the number of rotor blades or the airfoil of its blades, and its aerodynamic properties.

To simulate rotor's dynamics [1-6] and, in particular, its autorotation, there is a need to solve coupled aeroelastic problems. Such problems are complicated for numerical solution, since it is necessary to take into account interference between the flow and moving immersed body. So, the aim of this research is to develop an efficient numerical method for simulation of wind turbine rotors autorotation.

In case of massive body, when its average density is significantly higher than density of the flow, coupled aeroelastic problems can be solved using "step-by-step" weakcoupling numerical algorithm, firstly simulating flow around the body, which moves according to known parameters, and then computing the dynamics of the body under known hydrodynamic loads. Such case is considered in this research.
Immersed boundary methods [7] are suitable for numerical simulation in coupled aeroelastic problems, since they do not require a coincidence of cell edges and boundaries of the computational domain and allow solving problems when domain shape is irregular or it changes significantly in the simulation process due to aeroelastic body motion. The main advantage of these methods is that the mesh should not be reconstructed at every time step.

In the present study, the LS-STAG cut-cell immersed boundary method [8] is used for rotors autorotation simulation. This method permits solving problems on the Cartesian grid. The immersed boundary is represented with the levelset function [9]. Linear systems resulting from the LS-STAG discretization of the Navier-Stokes or Reynolds-averaged Navier-Stokes equations are solved using the BiCGStab method [10] with the ILU and Multigrid [11, 12] preconditioning. An original algorithm for the solver cost-coefficient estimation [13] is used for the optimal parameters of the multigrid preconditioner choice.

\section{Governing Equations}

The problem is considered in $2 \mathrm{D}$ unsteady case when the flow around a rigid airfoil is assumed to be viscous and 


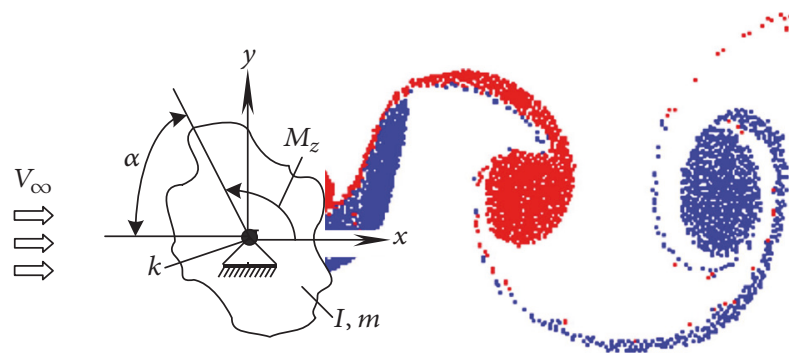

FIGURE 1: The airfoil of irregular shape with one rotational degree of freedom and schematic view of vortex wake behind it.

incompressible. The continuity and momentum equations are as follows:

$$
\begin{aligned}
\nabla \cdot \mathbf{v} & =0 \\
\frac{\partial \mathbf{v}}{\partial t}+(\mathbf{v} \cdot \nabla) \mathbf{v} & =\frac{1}{\rho} \nabla p+v \Delta \mathbf{v} .
\end{aligned}
$$

Here $\mathbf{v}$ is the velocity vector, $p$ is the pressure, $t$ is the time, $\rho$ is the flow density, and $v$ is the flow kinematic viscosity. The boundary conditions on the outer boundaries of the computational domain are as follows:

$$
\begin{aligned}
\left.\mathbf{v}\right|_{\text {inlet }} & =\mathbf{v}_{\infty}, \\
\left.\frac{\partial \mathbf{v}}{\partial \mathbf{n}}\right|_{\text {outlet }} & =0, \\
\left.\frac{\partial p}{\partial \mathbf{n}}\right|_{\text {inlet\&outlet }} & =0
\end{aligned}
$$

Here $\mathbf{v}_{\infty}$ is the velocity vector on the inlet boundary and $\mathbf{n}$ is the unit outer normal vector. The boundary conditions on the surface line of the airfoil are no-slip conditions:

$$
\begin{aligned}
\left.\mathbf{v}\right|_{\text {airfoil }} & =\mathbf{v}^{\mathrm{ib}}, \\
\left.\frac{\partial p}{\partial \mathbf{n}}\right|_{\text {airfoil }} & =0 .
\end{aligned}
$$

Here $\mathbf{v}^{\text {ib }}$ is the velocity of the immersed boundary.

To simulate the rotation of wind turbine rotors, the following dynamics equation is being solved:

$$
I \ddot{\alpha}+k \dot{\alpha}=M^{\text {flow }} .
$$

Here $\alpha$ is the rotation angle of the rotor, $I$ is the polar inertia moment of the rotor, $k$ is the viscous friction coefficient in the axis, and $M^{\text {flow }}$ is the aerodynamic torque. Two-dimensional problem is considered, so $M^{\text {flow }}=M_{z}$ (Figure 1).

\section{Numerical Method}

The Cartesian mesh with cells $\Omega_{i, j}=\left(x_{i-1}, x_{i}\right) \times\left(y_{j-1}, y_{j}\right)$ is introduced in the rectangular computational domain. It is considered that $\Gamma_{i, j}$ is the face of $\Omega_{i, j}$ cell and $\mathbf{x}_{i, j}^{c}=\left(x_{i}^{c}, y_{j}^{c}\right)$ is its center. Unknown components $u_{i, j}$ and $v_{i, j}$ of velocity

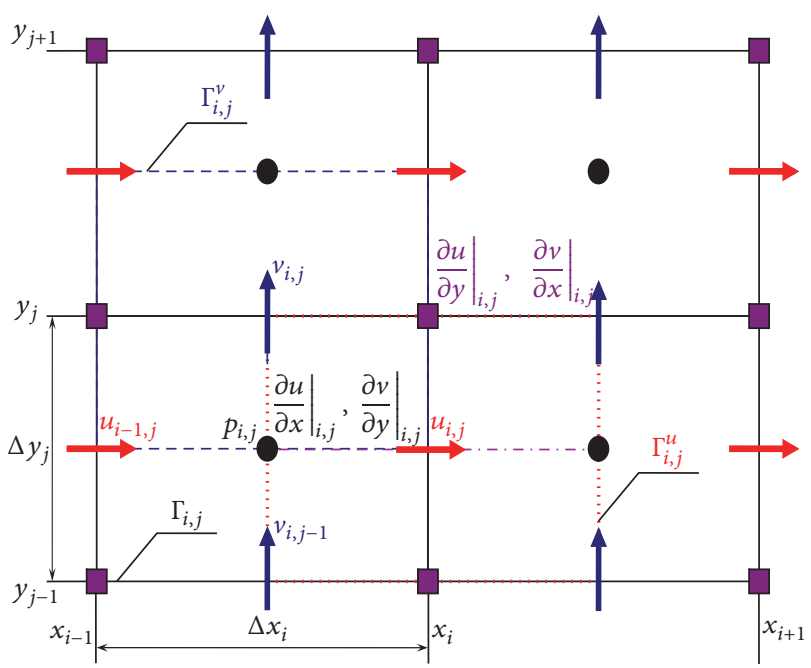

FIGURE 2: Staggered arrangement of the variables on the modified LS-STAG mesh.

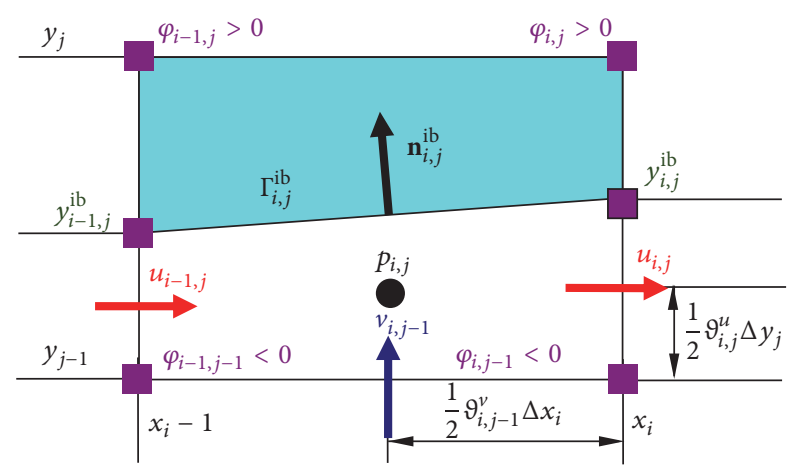

FIgURE 3: Example of a cut-cell.

vector $\mathbf{v}$ are computed in the middle of fluid parts of the cell faces. These points are the centers of control volumes $\Omega_{i, j}^{u}=\left(x_{i}^{c}, x_{i+1}^{c}\right) \times\left(y_{j-1}, y_{j}\right)$ and $\Omega_{i, j}^{v}=\left(x_{i-1}, x_{i}\right) \times\left(y_{j}^{c}, y_{j+1}^{c}\right)$ with faces $\Gamma_{i, j}^{u}$ and $\Gamma_{i, j}^{v}$, respectively (Figure 2). The main idea of this numerical method is described in our previous papers, for example, in [14].

Cells which the immersed boundary intersects are the socalled cut-cells (Figures 3 and 4). These cells contain the solid part together with the liquid one. The level-set function $\varphi$ is used for immersed boundary $\Gamma^{\mathrm{ib}}$ description. The boundary $\Gamma^{\mathrm{ib}}$ is represented by a line segment on the cut-cell $\Omega_{i, j}$. Locations of this segment's endpoints are defined by a linear interpolation of the variable $\varphi_{i, j}=\varphi\left(x_{i}, y_{j}\right)$.

A few notes should be mentioned about construction of the level-set function. The level-set function cannot be constructed analytically for rotor with complex shape, that is, Darrieus rotor, as for circular airfoil and for simple rotor shapes, for example, for Savonius rotor (Figures 5 and 6). For this reason, it is necessary to approximate the rotor surface line with a curve, which would make it possible to simulate both smooth parts of the boundary and the sharp edges. Moreover, it is desirable that the distance from an arbitrary 


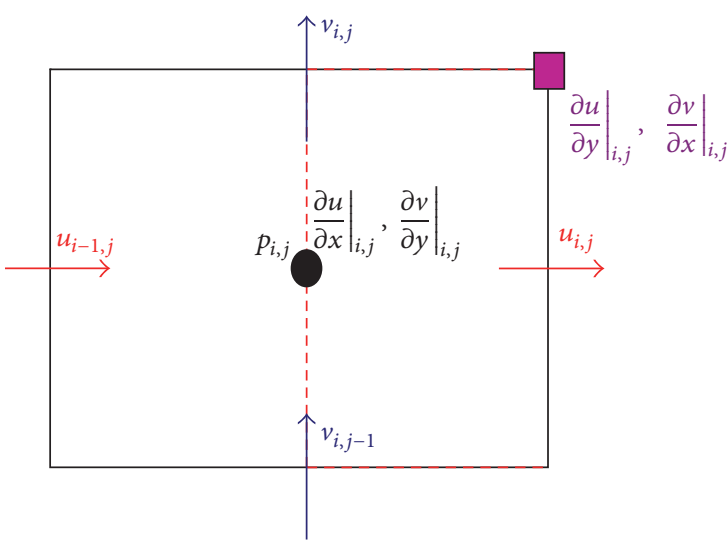

(a)

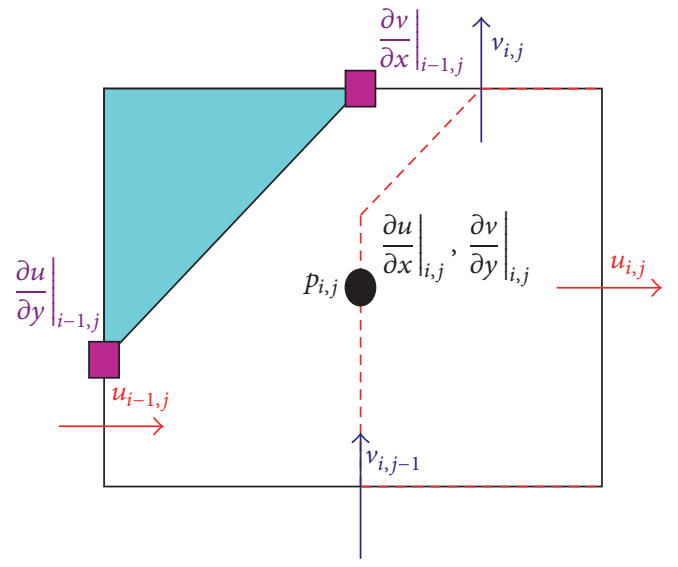

(c)

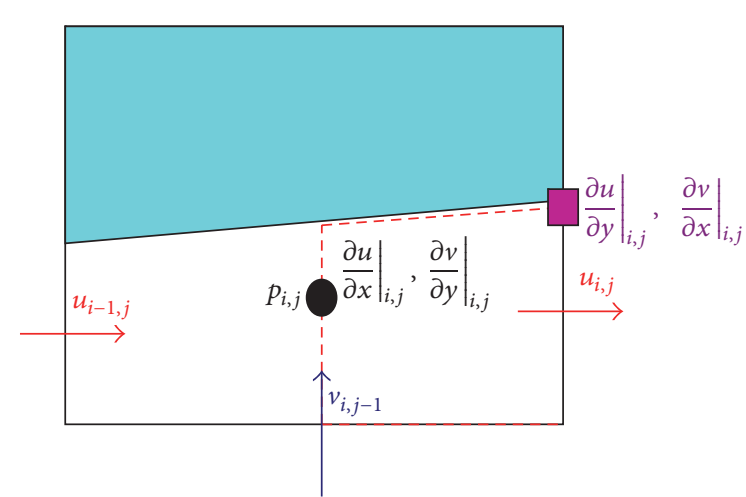

(b)

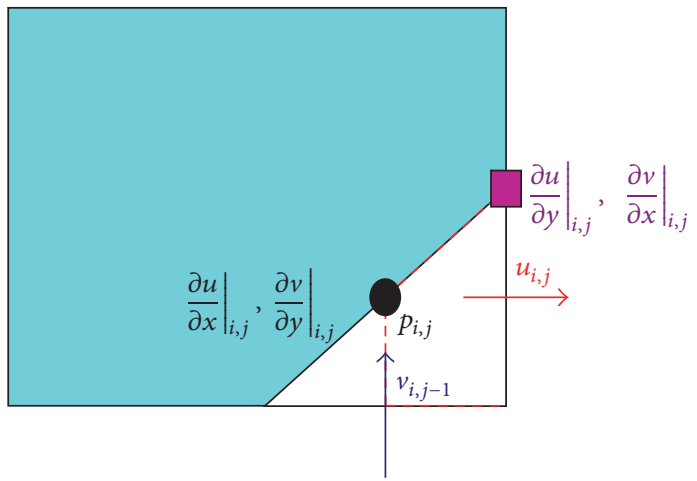

(d)

FIGURE 4: Locations of the variables discretization points in case of generic cells on the modified LS-STAG mesh: (a) Cartesian fluid cell; (b) north trapezoidal cell; (c) northwest pentagonal cell; (d) northwest triangle cell.

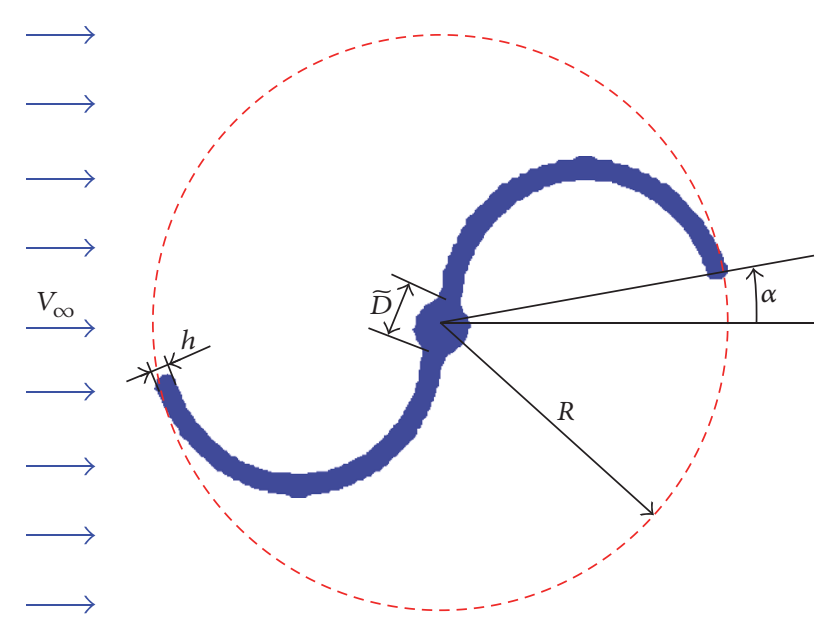

FIGURE 5: Schematic of the two-bucket Savonius rotor.

point to the boundary can be calculated easily. An efficient approach for the level-set function construction for an airfoil of arbitrary shape, which corresponds to the mentioned requirements, is described in [15]. The airfoil's surface line

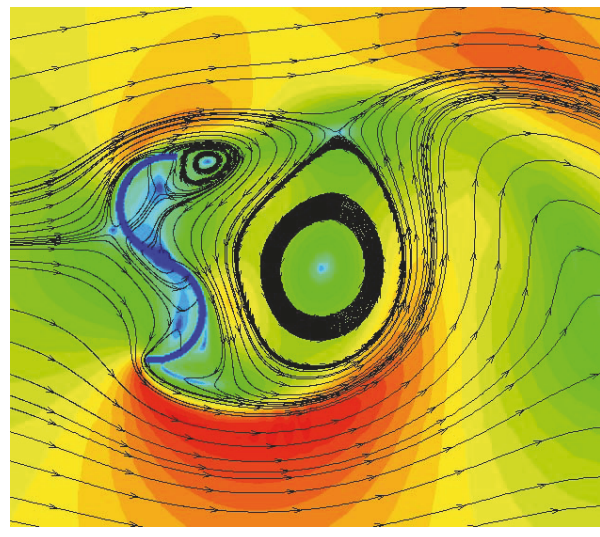

FIGURE 6: Flow past autorotating Savonius rotor with two blades.

approximation by using Bézier curve is taken as a basis of the developed algorithm.

In order to avoid the Bézier curve reconstruction at every time step for the airfoil surface line, the following approach can be used. At the beginning of the computation, the Bézier curve and its derivative should be built for the rotor blade 
airfoil at position $\alpha=0$. Then, the level-set function $\varphi^{n}$ in point $\left(x_{i}, y_{j}\right)$ can be computed as $\varphi^{n}\left(x_{i}, y_{j}\right)=\varphi^{0}\left(\widetilde{x_{i}}, \widetilde{y_{j}}\right)$ for the rotor rotated by angle $\alpha^{n}$ counterclockwise. Here $\varphi^{0}$ is the level-set function built for the rotor surface line at position $\alpha=0$ by using the Bézier curve and $\left(\widetilde{x_{i}}, \widetilde{y_{j}}\right)$ is the image of the point $\left(x_{i}, y_{j}\right)$ after clockwise rotation on the angle $\alpha^{n}$. Thus, the level-set function can be recalculated at any time without reconstructing the Bézier curve for each new rotor position.

The cell-face fraction ratios $\vartheta_{i, j}^{u}$ and $\vartheta_{i, j}^{v}$ are introduced. They take values in interval $[0,1]$ and represent the fluid parts of the east and north faces of $\Gamma_{i, j}$, respectively. Onedimensional linear interpolation of $\varphi\left(x_{i}, y\right)$ on the segment $\left[y_{j-1}, y_{j}\right]$ and $\varphi\left(x, y_{j}\right)$ on the segment $\left[x_{i-1}, x_{i}\right]$ is used for the cell-face fraction ratios computing:

$$
\begin{gathered}
\mathcal{\vartheta}_{i, j}^{u}=\frac{\min \left(\varphi_{i, j-1}, \varphi_{i, j}\right)}{\min \left(\varphi_{i, j-1}, \varphi_{i, j}\right)-\max \left(\varphi_{i, j-1}, \varphi_{i, j}\right)}, \\
\mathcal{\vartheta}_{i, j}^{v}=\frac{\min \left(\varphi_{i-1, j}, \varphi_{i, j}\right)}{\min \left(\varphi_{i-1, j}, \varphi_{i, j}\right)-\max \left(\varphi_{i-1, j}, \varphi_{i, j}\right)} .
\end{gathered}
$$

According to the concept of the LS-STAG method, normal Reynolds stress components are sampled on the base mesh (similar to pressure discretization) and shear ones are sampled in the upper right corners of the base mesh cells.

The time integration of the differential algebraic system resulting from continuity and momentum equations sampling in space is performed with a semi-implicit Euler scheme [8]. Predictor step leads to discrete analogues of the Helmholtz equation for velocities prediction at the next time layer. Corrector step leads to discrete analogue of the Poisson equation for pressure correction. After computation of the flow variables, the dynamics equation for the airfoil motion (4) should be solved.

The rotor angular velocity is $\omega=\dot{\alpha}$. So difference analogue of (4) can be written in the following form:

$$
I \frac{\omega^{n+1}-\omega^{n}}{\Delta t}+k \omega^{n}=M_{z}^{n} .
$$

The aerodynamic torque at the $n$-th time step can be computed as follows:

$$
\begin{aligned}
& M_{z}^{n} \\
& =\sum_{\text {Cut-cells } \Omega_{i, j}^{\text {ib }}}\left[\left.\left(x_{i}^{C}-X_{C}\right) F_{y a}^{h}\right|_{i, j} ^{n}-\left.\left(y_{j}^{C}-Y_{C}\right) F_{x a}^{h}\right|_{i, j} ^{n}\right] .
\end{aligned}
$$

Here $\left(x_{i}^{C}, y_{j}^{C}\right)$ are coordinates of the center of $\Omega_{i, j}$ cell, $\left(X_{C}, Y_{C}\right)$ are coordinates of the point around which the airfoil rotates, and $\left.F_{x a}^{h}\right|_{i, j} ^{n}$ and $\left.F_{y a}^{h}\right|_{i, j} ^{n}$ are the drag and lift forces acting on the solid part of the cut-cell $\Omega_{i, j}$ at the $n$-th time step:

$$
\begin{aligned}
\left.F_{x a}^{h}\right|_{i, j}= & {\left[n_{x} \Delta S\right]_{i, j}^{\mathrm{ib}}\left(p_{i, j}-\left.v \frac{\partial u}{\partial x}\right|_{i, j}\right) } \\
& -\nu \operatorname{Quad}_{i, j}^{\mathrm{ib}}\left(\frac{\partial u}{\partial y} \vec{e}_{y} \cdot \vec{n}\right),
\end{aligned}
$$

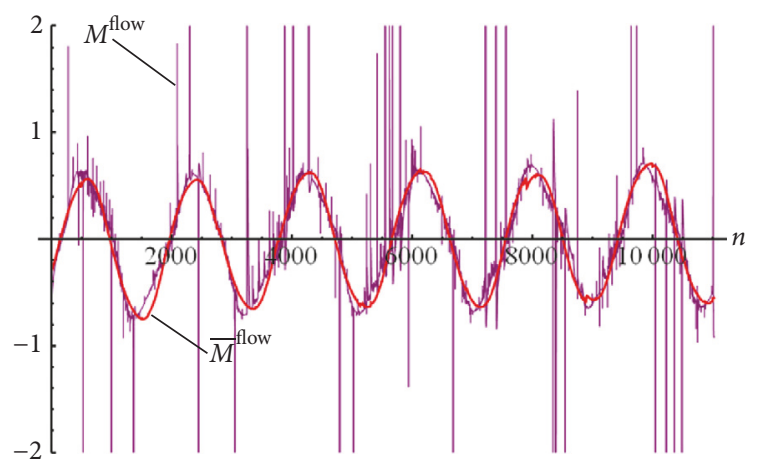

FIgURE 7: The time dependency for the torque $M^{\text {flow }}=M_{z}(t)$ (on the coarse grid $272 \times 292$ with time step $\Delta t=0.0001)$ and filtered time dependency $\bar{M}^{\text {flow }}=\bar{M}_{z}(t)$ for the torque.

$$
\begin{aligned}
\left.F_{y a}^{h}\right|_{i, j}= & -\nu \operatorname{Quad}_{i, j}^{\mathrm{ib}}\left(\frac{\partial v}{\partial x} \vec{e}_{x} \cdot \vec{n}\right) \\
& +\left[n_{y} \Delta S\right]_{i, j}^{\mathrm{ib}}\left(p_{i, j}-\left.v \frac{\partial v}{\partial y}\right|_{i, j}\right) .
\end{aligned}
$$

Here $\left[n_{x} \Delta S\right]_{i, j}^{\mathrm{ib}}=\left(\vartheta_{i-1, j}^{u}-\vartheta_{i, j}^{u}\right) \Delta y_{j},\left[n_{y} \Delta S\right]_{i, j}^{\mathrm{ib}}=\left(\vartheta_{i, j-1}^{v}-\vartheta_{i, j}^{v}\right) \Delta x_{i}$, $\Delta y_{j}=y_{j}-y_{j-1}, \Delta x_{i}=x_{i}-x_{i-1}$, and Quad ib are the quadratures of the shear stress which depend on the cut-cells [8].

The value of the rotor angular velocity at the next time step is computed from (6). New rotor position and the immersed boundary velocity can be defined by using this value:

$$
\begin{aligned}
& \alpha^{n+1}=\alpha^{n}+\omega^{n} \Delta t, \\
& \mathbf{v}_{i, j}^{\mathrm{ib}, n+1}=\left\{-\frac{y_{j}^{C}-Y_{C}}{\left|y_{j}^{C}-Y_{C}\right|} \cdot \omega^{n}\left|x_{i}^{C}-X_{C}\right|, \frac{x_{i}^{C}-X_{C}}{\left|x_{i}^{C}-X_{C}\right|}\right. \\
& \left.\cdot \omega^{n}\left|y_{j}^{C}-Y_{C}\right|\right\} .
\end{aligned}
$$

Such approach allows reconstructing the level-set function and all matrices and the source terms required for the next time step.

When investigating some complicated physical phenomenon, preliminary qualitative estimations for the considered construction behavior are very important. They allow finding domains for grid refinement, prediction of the structure's dynamic, and estimation of the CFL number. But large fluctuations in the aerodynamic loads can occur on the coarse grid. So the values of the torque acting on the airfoil should be filtered by the low-pass filter (Figure 7). For this purpose, a second-order Butterworth low-pass filter [16] is designed.

It is necessary to explain the reasons for choosing this filter. Filters with infinite impulse response are less expensive from a computational point of view than filters with finite impulse response. The frequency response of the Butterworth filter is maximally flat (i.e., has no ripples) in the passband 
and rolls off towards zero in the stopband [16]. Compared with a Chebyshev type I/type II filter or an elliptic filter, the Butterworth filter has a slower roll-off and thus will require a higher order to implement a particular stopband specification, but Butterworth filters have a more linear phase response in the passband than Chebyshev type I/type II and elliptic filters can achieve [16].

The transfer function of the Butterworth second-order filter is as follows:

$$
\begin{aligned}
H(s) & =\frac{1}{\left(s-(i-1) / \sqrt{2 \varepsilon_{p}}\right)\left(s+(i+1) / \sqrt{2 \varepsilon_{p}}\right)} \\
& =\frac{\varepsilon_{p}}{1+\sqrt{2 \varepsilon_{p}} s+\varepsilon_{p} s^{2}} .
\end{aligned}
$$

Here $s \in \mathbb{C}, i=\sqrt{-1}, \varepsilon_{p}=\sqrt{10^{R_{p} / 10}-1}$, and $R_{p}$ is a distortion level in the passband. As practice shows, filters of higher order can lead to the appearance of numerical instability in the filtered signal. For this function, $H(0)=\varepsilon_{p}$. Therefore the following transfer function corresponds to the normalized Butterworth second-order filter:

$$
H^{\mathrm{norm}}(s)=\frac{1}{1+\sqrt{2 \varepsilon_{p}} s+\varepsilon_{p} s^{2}} .
$$

In order to control the filter cutoff frequency, it is necessary to use the following transfer function:

$$
H_{\mathrm{LP}}(s)=H^{\text {norm }}\left(\frac{s}{\Omega_{x}}\right) .
$$

The sampling frequency of the torque is equal to $f_{d}=$ $1 / \Delta t$ in the numerical simulation ( $\Delta t$ is a time discretization step). It is necessary that the filter cut-off frequency is equal to $f_{s}=5 \mathrm{~Hz}$ and the suppression level on the cut-off frequency is equal to $R_{s}=3 \mathrm{~dB}$. So, the following condition imposed on the required filter frequency response function $\left|H_{\mathrm{LP}}(i \cdot \omega)\right|$ (Figure 8) must be satisfied for the required filter transfer function $H_{\mathrm{LP}}(s)$ :

$$
\begin{aligned}
\left|H_{\mathrm{LP}}\left(i \cdot \Omega_{s}\right)\right| & =\gamma=10^{-R_{s} / 20}, \\
\Omega_{s} & =\tan \left(\frac{\pi f_{s}}{f_{d}}\right)=\tan \left(\pi f_{s} \Delta t\right) .
\end{aligned}
$$

Solution of (13) leads to the following result:

$$
\begin{aligned}
\frac{1}{\sqrt{1+\left(\sqrt{\varepsilon_{p}} / \Omega_{x}\right)^{4} \Omega_{s}^{4}}}=\gamma \Longleftrightarrow \\
\frac{\sqrt{\varepsilon_{p}}}{\Omega_{x}}=\frac{\sqrt[4]{1-\gamma^{2}}}{\sqrt{\gamma} \Omega_{s}}=\xi .
\end{aligned}
$$

Thus, the desired filter transfer function can be written as follows:

$$
H_{\mathrm{LP}}(s)=\frac{1}{1+\sqrt{2} \xi_{s}+(\xi s)^{2}} .
$$

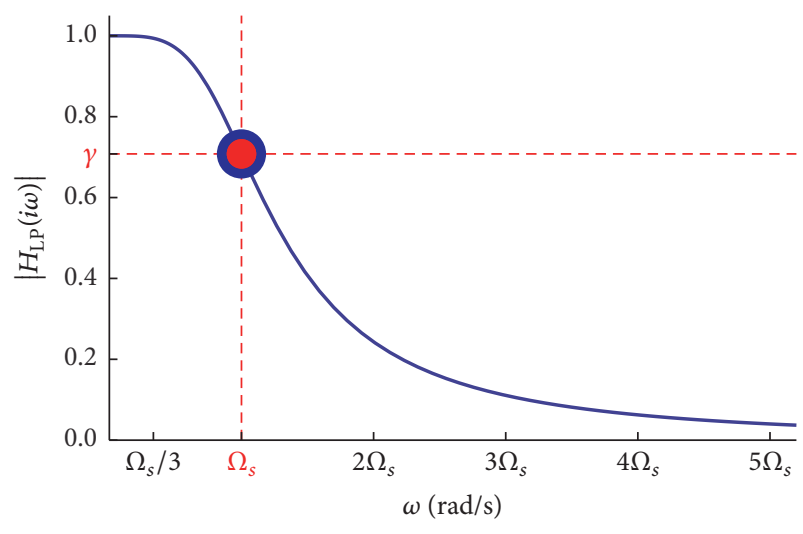

FIGURE 8: The required filter frequency response function.

To obtain the corresponding digital filter coefficients, there is a need to use the bilinear transformation [16]:

$$
\begin{aligned}
& H_{\mathrm{LP}}\left(\frac{1-z^{-1}}{1+z^{-1}}\right) \\
& =\frac{1+2 z^{-1}+z^{-2}}{\left(1+\sqrt{2} \xi+\xi^{2}\right)+2\left(1-\xi^{2}\right) z^{-1}+\left(1-\sqrt{2} \xi+\xi^{2}\right) z^{-2}} .
\end{aligned}
$$

Here $z \in \mathbb{R}$. It is known that

$$
H_{\mathrm{LP}}\left(\frac{1-z^{-1}}{1+z^{-1}}\right)=\frac{b_{0}+b_{1} z^{-1}+b_{2} z^{-2}}{1+a_{1} z^{-1}+a_{2} z^{-2}}
$$

for the second-order low-pass filter with infinite impulse response. Therefore, it can be obtained from formulae (16) and (17) that the designed digital filter coefficients are the following:

$$
\begin{aligned}
& b_{0}=b_{2}=\frac{1}{1+\sqrt{2} \xi+\xi^{2}}, \\
& b_{1}=\frac{2}{1+\sqrt{2} \xi+\xi^{2}}, \\
& a_{1}=\frac{2\left(1-\xi^{2}\right)}{1+\sqrt{2} \xi+\xi^{2}}, \\
& a_{2}=\frac{1-\sqrt{2} \xi+\xi^{2}}{1+\sqrt{2} \xi+\xi^{2}} .
\end{aligned}
$$

So, the filtered value of the torque acting on the airfoil from the flow at the time $t_{n+1}=(n+1) \Delta t$ is

$$
\begin{aligned}
\bar{M}_{z}^{n+1}= & b_{0} \cdot M_{z}^{n+1}+b_{1} \cdot M_{z}^{n}+b_{2} \cdot M_{z}^{n-1} \\
& -\left(a_{1} \cdot \bar{M}_{z}^{n}+a_{2} \cdot \bar{M}_{z}^{n-1}\right) .
\end{aligned}
$$

\section{Numerical Experiments}

We considered autorotation of Savonius rotor with two blades (Figure 5) at $\operatorname{Re}=1.96 \cdot 10^{5}$. The radius of rotation $R$ was 
measured from the axis of rotation to the outer edge of the buckets. The turbine-swept area $A_{s}$ is equal to $\pi R^{2}$. Reynolds number is based on rotor diameter. The following parameters were used in the simulation:

$$
\begin{aligned}
V_{\infty} & =1.0, \\
R & =\frac{D}{2}=1.0, \\
\widetilde{D} & =0.1 D, \\
h & =0.05 R, \\
k & =0.0, \\
I & =10.0, \\
\alpha_{0} & =\alpha(0)=\frac{\pi}{18} .
\end{aligned}
$$

It should be noted that the above-proposed algorithm for level-set reconstruction can be easy to apply for rotors of other shapes, that is, Darrieus rotor.

The dimensionless average rotor angular velocity is in the following range:

$$
\bar{\omega}=0.4, \ldots, 1.6
$$

Averaging was carried out over 16 dimensionless time units. This time is enough for the rotor to make full turn with the smallest value of average rotor angular velocity $\bar{\omega}=0.4$. At the chosen values of the parameters, tip speed ratio is equal to

$$
X_{\infty}=\frac{R \bar{\omega}}{V_{\infty}}=\bar{\omega}
$$

torque coefficient value $\bar{C}_{Q}$ is obtained by averaging of nonstationary dependency $C_{Q}(t)$ over time, where

$$
C_{Q}(t)=\frac{2 \bar{M}_{z}(t)}{\rho V_{\infty}^{2} R A_{s}}=\frac{2 \bar{M}_{z}(t)}{\pi} .
$$

Similarly, the power coefficient $\bar{C}_{P}$ is obtained by averaging of nonstationary dependency $C_{P}(t)$ over time, where

$$
C_{P}(t)=\frac{2 \bar{M}_{z}(t) \bar{\omega}}{\rho V_{\infty}^{3} A_{s}}=\frac{2 \bar{M}_{z}(t) \bar{\omega}}{\pi} .
$$

A number of computations have been performed on nonuniform grid $544 \times 496$. The uniform mesh block with space discretization step $\Delta h=D / 128$ was used in the vicinity of the rotor. Time discretization step was equal to $\Delta t=10^{-5}$. Computations were performed on a server based on the Intel C610 platform using the Intel Xeon E5-1620 V3 4-core processor (3.5 GHz) with Hyper-Threading support (8 logical cores). The server is equipped with 16 GB of ECC DDR4-2133 RAM and two hard drives (2 TB), united in a RAID1 disk volume. This server is running Windows Server 2012 R2 operating system. To simulate 1 dimensionless time unit, about 24 hours are required.

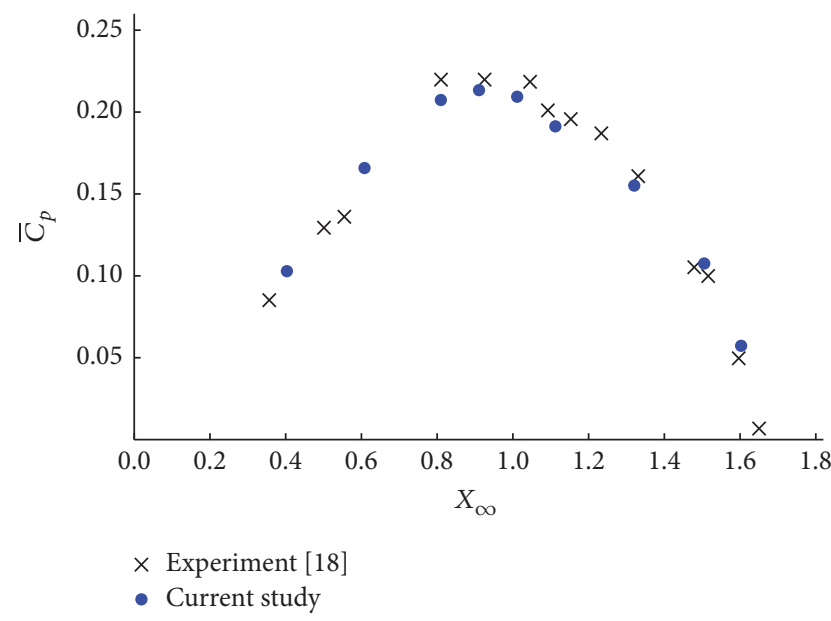

FIgURE 9: Comparison of computed power coefficients $\bar{C}_{P}$ for a two-bucket Savonius rotor at $\mathrm{Re}=1.96 \cdot 10^{5}$ with experimental data (Sheldahl et al. [17] at $\mathrm{Re}=3.9 \cdot 10^{5}$ and Shankar [18] at $\left.\operatorname{Re}=1.96 \cdot 10^{5}\right)$.

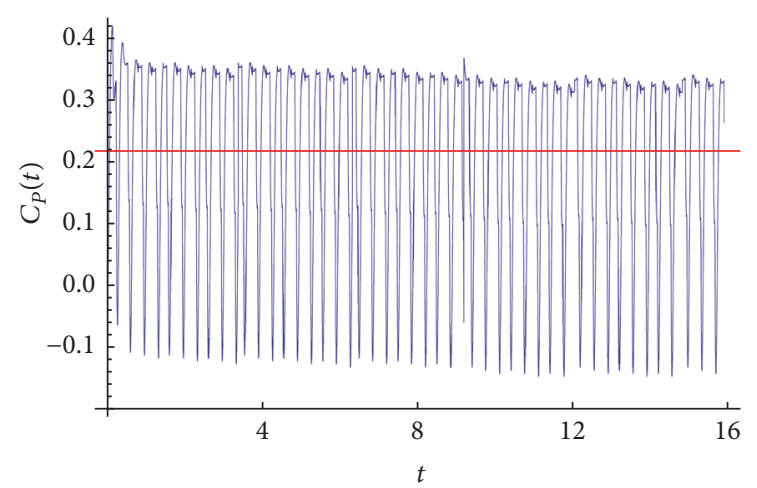

FIgURE 10: Time evolution of the power coefficient $C_{p}(t)$ at $\operatorname{Re}=$ $1.96 \cdot 10^{5}$ and $\bar{\omega}=0.9$. The red line corresponds to the average value $\bar{C}_{P}$.

The computed values of the power coefficient $\bar{C}_{P}$ were compared with the experimental data $[17,18]$ presented in [18]. As can be seen from Figure 9, the computational results are in good agreement with experiment. An example of simulated nonstationary dependency $C_{P}(t)$ and computed value of the corresponding power coefficient $\bar{C}_{P}$ at $\operatorname{Re}=1.96 \cdot 10^{5}$ and $\bar{\omega}=0.9$ is shown in Figure 10.

\section{Conclusions}

We have the following conclusions

(i) A software package is developed for numerical simulation of wind turbine rotors autorotation by using the modified LS-STAG level-set/cut-cell immersed boundary method. This software package can be used for rotors of other shapes (Savonius rotor, Darrieus rotor, etc.).

(ii) Algorithms for level-set function construction and recalculation are described. 
(iii) A second-order Butterworth low-pass filter is designed for aerodynamic torque filtrating at simulation on the coarse grid.

(iv) Simulation of flow past autorotating Savonius rotor with two blades is considered at $\operatorname{Re}=1.96 \cdot 10^{5}$.

(v) Computational results are in good qualitative agreement with the experimental data.

\section{Conflicts of Interest}

The authors declare that there are no conflicts of interest regarding the publication of this paper.

\section{Acknowledgments}

This research is partially supported by Russian Ministry of Education and Science (Project no. 9.2422.2017/PP) and Russian Federation President Grant for Young Russian Ph.D. Scientists (Project no. MK-7431.2016.8).

\section{References}

[1] P.-A. Krogstad and J. A. Lund, "An experimental and numerical study of the performance of a model turbine," Wind Energy, vol. 15, no. 3, pp. 443-457, 2012.

[2] N. N. Sorensen, J. A. Michelsen, and S. Schreck, "Navier-stokes predictions of the NREL phase VI rotor in the NASA Ames 80-by-120 wind tunnel," in Proceedings of the 2002 ASME Wind Energy Symposium, pp. 94-105, Reno, Nev, USA, January 2002.

[3] K. Rogowski and R. Maroński, "CFD computation of the savonius rotor," Journal of Theoretical and Applied Mechanics, vol. 53, no. 1, pp. 37-45, 2015.

[4] R. Gupta and K. K. Sharma, "Flow physics of a combined Darrieus-Savonius rotor using computational fluid dynamics (CFD)," Int. Research J. Eng. Science, Technology and Innovation, vol. 1, pp. 1-13, 2012.

[5] L. Oggiano, "CFD Simulations on the NTNU Wind Turbine Rotor and Comparison with Experiments," in Proceedings of the Renewable Energy Research Conference, RERC 2014, pp. 111-116, June 2014.

[6] M. H. Mohamed, A. M. Ali, and A. A. Hafiz, "CFD analysis for H-rotor Darrieus turbine as a low speed wind energy converter," Engineering Science and Technology, an International Journal, vol. 18, no. 1, pp. 1-13, 2015.

[7] R. Mittal and G. Iaccarino, "Immersed boundary methods," in Annual review of fluid mechanics. Vol. 37, vol. 37 of Annu. Rev. Fluid Mech., pp. 239-261, Annual Reviews, Palo Alto, CA, 2005.

[8] Y. Cheny and O. Botella, "The LS-STAG method: A new immersed boundary/level-set method for the computation of incompressible viscous flows in complex moving geometries with good conservation properties," Journal of Computational Physics, vol. 229, no. 4, pp. 1043-1076, 2010.

[9] S. Osher and R. Fedkiw, Level Set Methods and Dynamic Implicit Surfaces, vol. 153, Springer, New York, NY, USA, 2003.

[10] H. A. van der Vorst, "BI-CGSTAB: a fast and smoothly converging variant of BI-CG for the solution of nonsymmetric linear systems," SIAM Journal on Scientific and Statistical Computing, vol. 13, no. 2, pp. 631-644, 1992.

[11] P. Wesseling, An Introduction to Multigrid Methods, John Wiley \& Sons, Chichester, UK, 1992.
[12] J. van Kan, C. Vuik, and P. Wesseling, "Fast pressure calculation for 2D and 3D Time dependent incompressible flow," Numerical Linear Algebra with Applications, vol. 7, no. 6, pp. 429-447, 2000.

[13] I. Marchevsky and V. Puzikova, "OpenFOAM iterative methods efficiency analysis for linear systems solving," Proceedings of the Institute for System Programming of RAS, vol. 24, pp. 71-86, 2013.

[14] V. V. Puzikova and I. K. Marchevsky, "Application of the LSSTAG immersed boundary method for numerical simulation in coupled aeroelastic problems," in Proceedings of the Joint 11th World Congress on Computational Mechanics, WCCM 2014, the 5th European Conference on Computational Mechanics, ECCM 2014 and the 6th European Conference on Computational Fluid Dynamics, ECFD 2014, pp. 1995-2006, esp, July 2014.

[15] V. V. Puzikova, "Construction of Level-Set Function for an Airfoil of Arbitrary Topology when Modelling a Flow past It Using the LS-STAG Method," in Herald of the Bauman Moscow State Technical University. Natural Sciences, pp. 163-173, 2012.

[16] R. G. Lyons, Understanding Digital Signal Processing, Pearson Education, 2010.

[17] R. E. Sheldahl, B. F. Blackwell, and L. V. Feltz, "Wind tunnel performance data for two- and three-bucket savonius rotors," J Energy, vol. 2, no. 3, pp. 160-164, 1978.

[18] P. N. Shankar, National Aeronautical Laboratory, AE-TM-3-76, National Aeronautical Laboratory, Bangalore, India, 1976. 


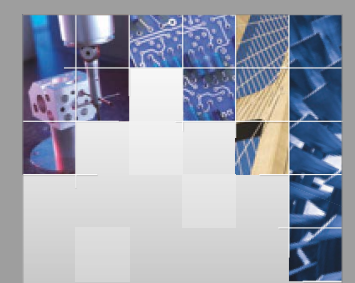

\section{Enfincering}
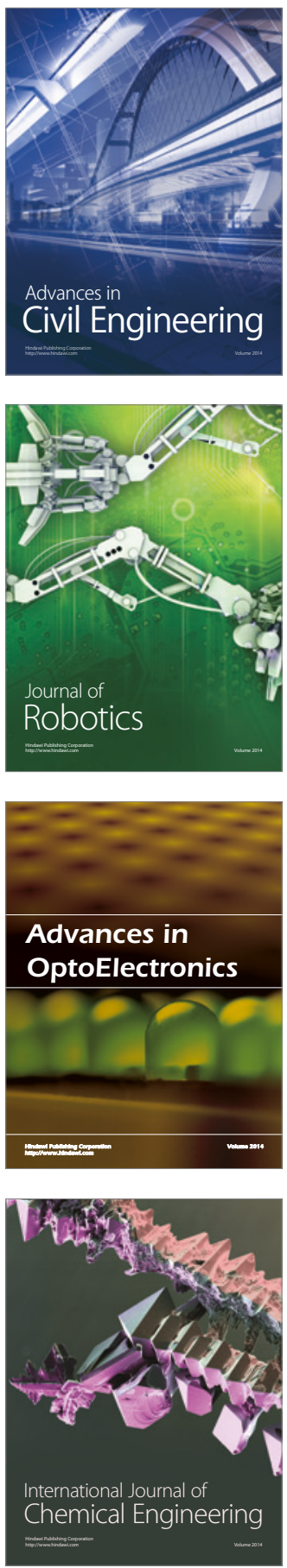

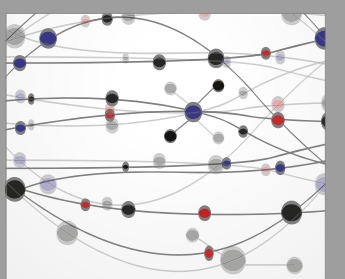

The Scientific World Journal

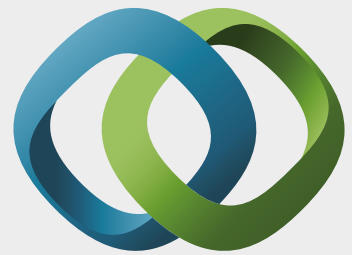

\section{Hindawi}

Submit your manuscripts at

https://www.hindawi.com
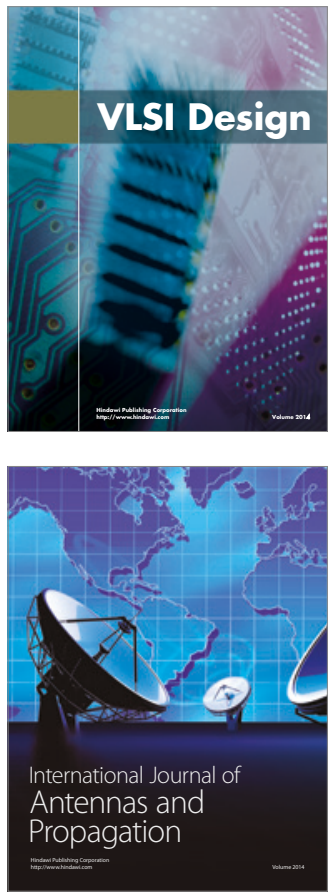

\section{Rotating}

Machinery
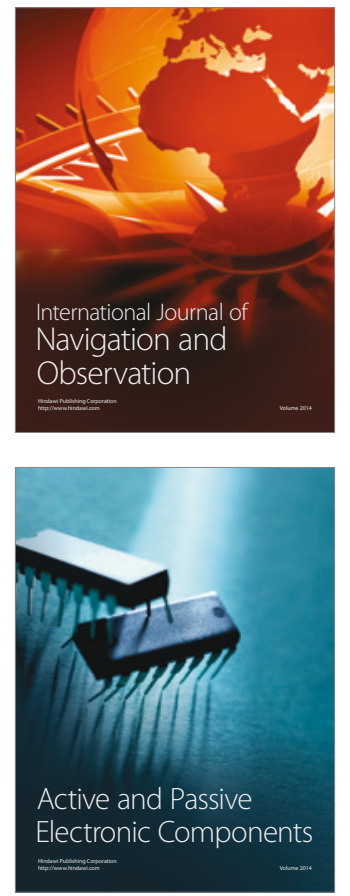
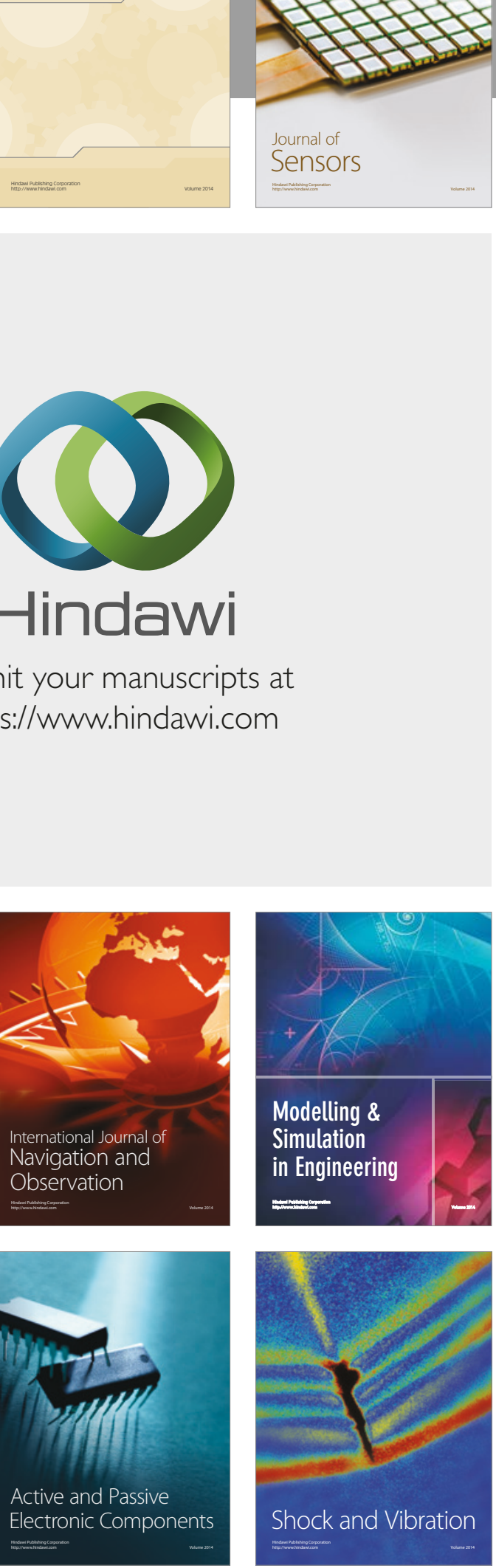
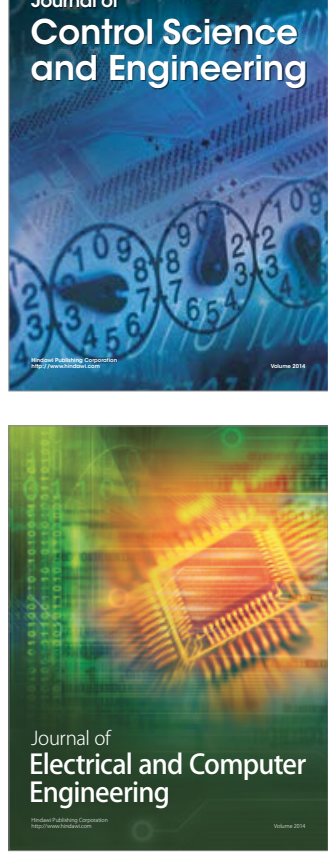

Distributed

Journal of

Control Science

and Engineering
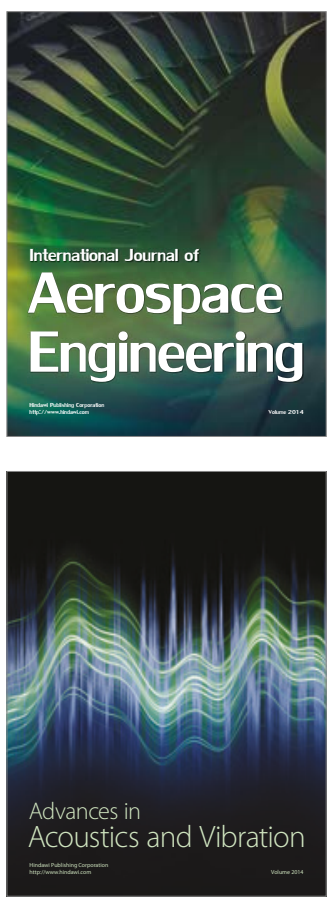

Sensor Networks 